ORIGINAL ARTICLE

\section{Survival in BRAF V600-Mutant Advanced Melanoma Treated with Vemurafenib}

\author{
Jeffrey A. Sosman, M.D., Kevin B. Kim, M.D., Lynn Schuchter, M.D., \\ Rene Gonzalez, M.D., Anna C. Pavlick, D.O., Jeffrey S. Weber, M.D., Ph.D., \\ Grant A. McArthur, M.B., B.S., Ph.D., Thomas E. Hutson, D.O., \\ Stergios J. Moschos, M.D., Keith T. Flaherty, M.D., Peter Hersey, F.R.A.C.P., D.Phil., \\ Richard Kefford, M.B., B.S., Ph.D., Donald Lawrence, M.D., Igor Puzanov, M.D., \\ Karl D. Lewis, M.D., Ravi K. Amaravadi, M.D., Bartosz Chmielowski, M.D., Ph.D., \\ H. Jeffrey Lawrence, M.D., Yu Shyr, Ph.D., Fei Ye, Ph.D., Jiang Li, Ph.D., \\ Keith B. Nolop, M.D., Richard J. Lee, M.D., Andrew K. Joe, M.D., \\ and Antoni Ribas, M.D., Ph.D.
}

A BSTRACT

\section{BACKGROUND}

Approximately 50\% of melanomas harbor activating (V600) mutations in the serinethreonine protein kinase B-RAF (BRAF). The oral BRAF inhibitor vemurafenib (PLX4032) frequently produced tumor regressions in patients with BRAF V600mutant metastatic melanoma in a phase 1 trial and improved overall survival in a phase 3 trial.

\section{METHODS}

We designed a multicenter phase 2 trial of vemurafenib in patients with previously treated BRAF V600-mutant metastatic melanoma to investigate the efficacy of vemurafenib with respect to overall response rate (percentage of treated patients with a tumor response), duration of response, and overall survival. The primary end point was the overall response rate as ascertained by the independent review committee; overall survival was a secondary end point.

\section{RESULTS}

A total of 132 patients had a median follow-up of 12.9 months (range, 0.6 to 20.1). The confirmed overall response rate was 53\% (95\% confidence interval [CI], 44 to 62 ; $6 \%$ with a complete response and $47 \%$ with a partial response), the median duration of response was 6.7 months ( $95 \% \mathrm{CI}, 5.6$ to 8.6), and the median progression-free survival was 6.8 months $(95 \% \mathrm{CI}, 5.6$ to 8.1). Primary progression was observed in only $14 \%$ of patients. Some patients had a response after receiving vemurafenib for more than 6 months. The median overall survival was 15.9 months ( $95 \%$ CI, 11.6 to 18.3). The most common adverse events were grade 1 or 2 arthralgia, rash, photosensitivity, fatigue, and alopecia. Cutaneous squamous-cell carcinomas (the majority, keratoacanthoma type) were diagnosed in $26 \%$ of patients.

\section{CONCLUSIONS}

Vemurafenib induces clinical responses in more than half of patients with previously treated BRAF V600-mutant metastatic melanoma. In this study with a long follow-up, the median overall survival was approximately 16 months. (Funded by Hoffmann-La Roche; ClinicalTrials.gov number, NCT00949702.)
From the Vanderbilt-Ingram Cancer Center (J.A.S., I.P.) and the Center for Quantitative Sciences, Vanderbilt University Medical Center (Y.S., F.Y.) - both in Nashville; the University of Texas M.D. Anderson Cancer Center, Houston (K.B.K.); Abramson Cancer Center at the University of Pennsylvania, Philadelphia (L.S., R.K.A.); the University of Colorado, Denver (R.G., K.D.L.); New York University Medical Center, New York (A.C.P.); Moffitt Cancer Center, Tampa, FL (J.S.W.); Peter MacCallum Cancer Centre, East Melbourne, VIC (G.A.M.), the University of Newcastle, Newcastle, NSW (P.H.), and Westmead Institute for Cancer Research and Melanoma Institute Australia, Westmead, NSW (R.K.) - all in Australia; Texas OncologyBaylor Sammons Cancer Center, Dallas (T.E.H.); the University of Pittsburgh, Pittsburgh (S.J.M.); Massachusetts General Hospital Cancer Center, Boston (K.T.F., D.L.); the Jonsson Comprehensive Cancer Center at the University of California, Los Angeles (B.C., A.R.), Roche Molecular Systems, Pleasanton (H.J.L.), and Plexxikon, Berkeley (K.B.N.) - all in California; and Hoffmann-La Roche, Nutley, NJ (J.L., R.J.L., A.K.J.). Address reprint requests to Dr. Sosman at VanderbiltIngram Cancer Center, 777 Preston Research Bldg., Nashville, TN 37232-6307, or at jeff.sosman@vanderbilt.edu.

N Engl J Med 2012;366:707-14.

Copyright @ 2012 Massachusetts Medical Society. 
P ATIENTS WITH METASTATIC MELANOMA have a median survival of 6 to 10 months. ${ }^{1-5}$ Few patients have a response to systemic therapies. ${ }^{1,6}$ Ipilimumab, a monoclonal antibody that blocks cytotoxic T-lymphocyte-associated antigen 4 (CTLA4) on lymphocytes, has recently been associated with superior overall survival, with median overall survival of 10.1 months among previously treated patients and 11.2 months among previously untreated patients. ${ }^{7,8}$ However, the majority of patients do not have a response to antiCTLA4 antibody therapy and still need effective therapeutic options.

In 2002, investigators at the Sanger Institute discovered that mutations in the gene encoding the serine-threonine protein kinase B-RAF (BRAF) occurred in more than $60 \%$ of melanomas initially tested. ${ }^{9}$ Melanomas carrying a BRAF V600E mutation constitutively activate the mitogen-activated protein kinase (MAPK) pathway, promoting cell proliferation and preventing apoptosis. ${ }^{10}$ Vemurafenib (PLX4032) was developed as a potent kinase inhibitor with specificity for the BRAF V600E mutation within cancer cells. ${ }^{11-14}$

A phase 1 trial of escalating doses of vemurafenib identified a recommended phase 2 dose of $960 \mathrm{mg}$ orally, twice daily, that was subsequently tested in an extension cohort of 32 patients with BRAF V600-mutant metastatic melanoma. ${ }^{15}$ Twenty-six of 32 patients (81\%) had an objective response (56\% with a confirmed response).

To determine the rate of response to vemurafenib, we conducted a phase 2 trial in patients with previously treated BRAF V600-mutant metastatic melanoma with central review of confirmed responses by an independent review committee (IRC). After enrollment was completed, results of a phase 3 trial (BRAF Inhibitor in Melanoma 3 [BRIM-3]; ClinicalTrials.gov number, NCT01006980) of vemurafenib versus dacarbazine chemotherapy in untreated BRAF V600mutant metastatic melanoma were published. ${ }^{16}$ The phase 3 trial showed significant improvement in both progression-free survival and overall survival with vemurafenib over chemotherapy, with hazard ratios of 0.26 and 0.37 , respectively, in an early interim analysis of overall survival. The median duration of follow-up was slightly less than 4 months, inadequate to address longterm outcomes with vemurafenib. Our phase 2 trial had a much longer follow-up period.

\section{METHODS}

\section{STUDY DESIGN}

In this multicenter phase 2 clinical trial, we enrolled patients with previously treated metastatic melanoma bearing a BRAF V600 mutation, as detected with a polymerase-chain-reaction (PCR)based test. Patients received vemurafenib at a dose of $960 \mathrm{mg}$ orally twice daily until the development of unacceptable toxic effects or disease progression. Patients with disease progression were permitted to continue vemurafenib if the investigator believed the patient would benefit clinically.

The protocol and the statistical analysis plan are available with the full text of this article at NEJM.org. The protocol was approved by the institutional review board at each participating institution, and the study was conducted in accordance with the protocol and the ethical principles of the Declaration of Helsinki. All study participants provided written informed consent.

The trial was designed jointly by the senior academic authors and representatives of the sponsor, Hoffmann-La Roche. Data were collected by the sponsor and analyzed in collaboration with the senior academic authors, who along with all coauthors vouch for the completeness and accuracy of the data and analyses and for the conformance of this report to the protocol, as amended. The corresponding academic author prepared an initial draft of the manuscript. All authors contributed to subsequent drafts and made the decision to submit the manuscript for publication.

\section{ELIGIBILITY CRITERIA}

Eligibility criteria included an age of 18 years or older, histologically proven stage IV melanoma, progressive disease after at least one prior systemic treatment for advanced disease (including interleukin-2 or standard chemotherapy), an Eastern Cooperative Oncology Group (ECOG) performance status score ${ }^{17}$ of 0 or 1 (where 0 means fully active, able to carry on all predisease performance without restriction, and 1 means restricted from physically strenuous activity but ambulatory and able to carry out work of a light or sedentary nature), brain metastasis controlled for at least 3 months after completion of local therapy, no other invasive cancer within 5 years before enrollment, and adequate hematologic, hepatic, and renal function. 
BRAF V600 MUTATION ANALYSIS

Mutation status was determined by means of a realtime PCR assay (Cobas 4800 BRAF V600 Mutation Test, Roche Molecular Systems) (see the Methods section in the Supplementary Appendix, available at NEJM.org). DNA was subsequently retested with a validated two-fold bidirectional Sanger sequencing method at a central laboratory. Samples with Sanger sequencing results that were invalid or discordant from the PCR result or that were identified as non-V600E mutations were subjected to a massively parallel pyrosequencing method (454 GS FLX Titanium, 454 Life Sciences). ${ }^{18}$

\section{TUMOR ASSESSMENTS}

Patients underwent baseline tumor imaging, including screening with the use of magnetic resonance imaging (MRI) or contrast-enhanced computed tomography (CT) of the brain, within 28 days before the first dose of the study drug was administered. Tumor assessments were performed every 6 weeks and at the final visit. Blinded IRC assessments of response to therapy were conducted either at the time of disease progression or at scheduled time points. Tumor assessments by both the IRC and investigators were performed according to Response Evaluation Criteria in Solid Tumors (RECIST), version 1.1 (Table A in the Supplementary Appendix). ${ }^{19}$

\section{MANAGEMENT OF TOXIC EFFECTS}

Toxic effects were graded according to the Common Terminology Criteria for Adverse Events (CTCAE), version 4.0. Toxic effects were managed generally by interrupting treatment until improving to grade 1 or to baseline status, with subsequent dose reductions required in some patients. Doses were reduced to $720 \mathrm{mg}$ or $480 \mathrm{mg}$ twice daily, depending on the severity of the event. Dermatologic evaluations for cutaneous squamous-cell carcinoma were performed regularly, and any lesions found were surgically excised. Although this disease is considered a grade 3 toxic effect according to the CTCAE, affected patients were allowed to continue treatment without dose interruption or reduction.

\section{STATISTICAL ANALYSIS}

The primary efficacy end point was the overall response rate as assessed by the IRC. The overall response rate was defined as the number of pa-

\begin{tabular}{|c|c|}
\hline Characteristic & Value \\
\hline \multicolumn{2}{|l|}{ Sex-no. (\%) } \\
\hline Female & $51(39)$ \\
\hline Male & $81(61)$ \\
\hline \multicolumn{2}{|c|}{ Race or ethnic group — no. (\%) † } \\
\hline White & $130(98)$ \\
\hline Hispanic & $2(2)$ \\
\hline \multicolumn{2}{|l|}{ Age } \\
\hline Median - yr & 51.5 \\
\hline$<65 \mathrm{yr}-$ no. (\%) & $107(81)$ \\
\hline$\geq 65$ yr - no. (\%) & $25(19)$ \\
\hline \multicolumn{2}{|c|}{ No. of prior therapies - no. (\%) } \\
\hline 1 & $67(51)$ \\
\hline 2 & $36(27)$ \\
\hline$\geq 3$ & $29(22)$ \\
\hline \multicolumn{2}{|c|}{ Previous interleukin-2 - no. (\%) } \\
\hline No & $81(61)$ \\
\hline Yes & $51(39)$ \\
\hline \multicolumn{2}{|c|}{ Previous ipilimumab — no. (\%) } \\
\hline Yes & $7(5)$ \\
\hline No & $125(95)$ \\
\hline \multicolumn{2}{|c|}{ ECOG status score - no. (\%) } \\
\hline 0 & $61(46)$ \\
\hline 1 & $71(54)$ \\
\hline \multicolumn{2}{|c|}{ Metastatic stage at diagnosis - no. (\%) } \\
\hline $\mathrm{Mla}$ & $33(25)$ \\
\hline Mlb & $18(14)$ \\
\hline $\mathrm{Mlc}$ & $81(61)$ \\
\hline \multicolumn{2}{|l|}{ Serum LDH - no. (\%) } \\
\hline Normal & $67(51)$ \\
\hline Elevated & $65(49)$ \\
\hline
\end{tabular}

* ECOG denotes Eastern Cooperative Oncology Group, and LDH lactate dehydrogenase.

$\uparrow$ Race or ethnic group was determined by the research staff.

tients with a complete or partial response divided by the total number of treated patients. The response (the change in tumor measurement from baseline) had to be confirmed with at least one repeat tumor assessment performed sequentially at least 28 days after the criterion for response was first met. The overall response rate and exact twosided $95 \%$ confidence interval were calculated by means of the Clopper-Pearson method. ${ }^{20}$ 


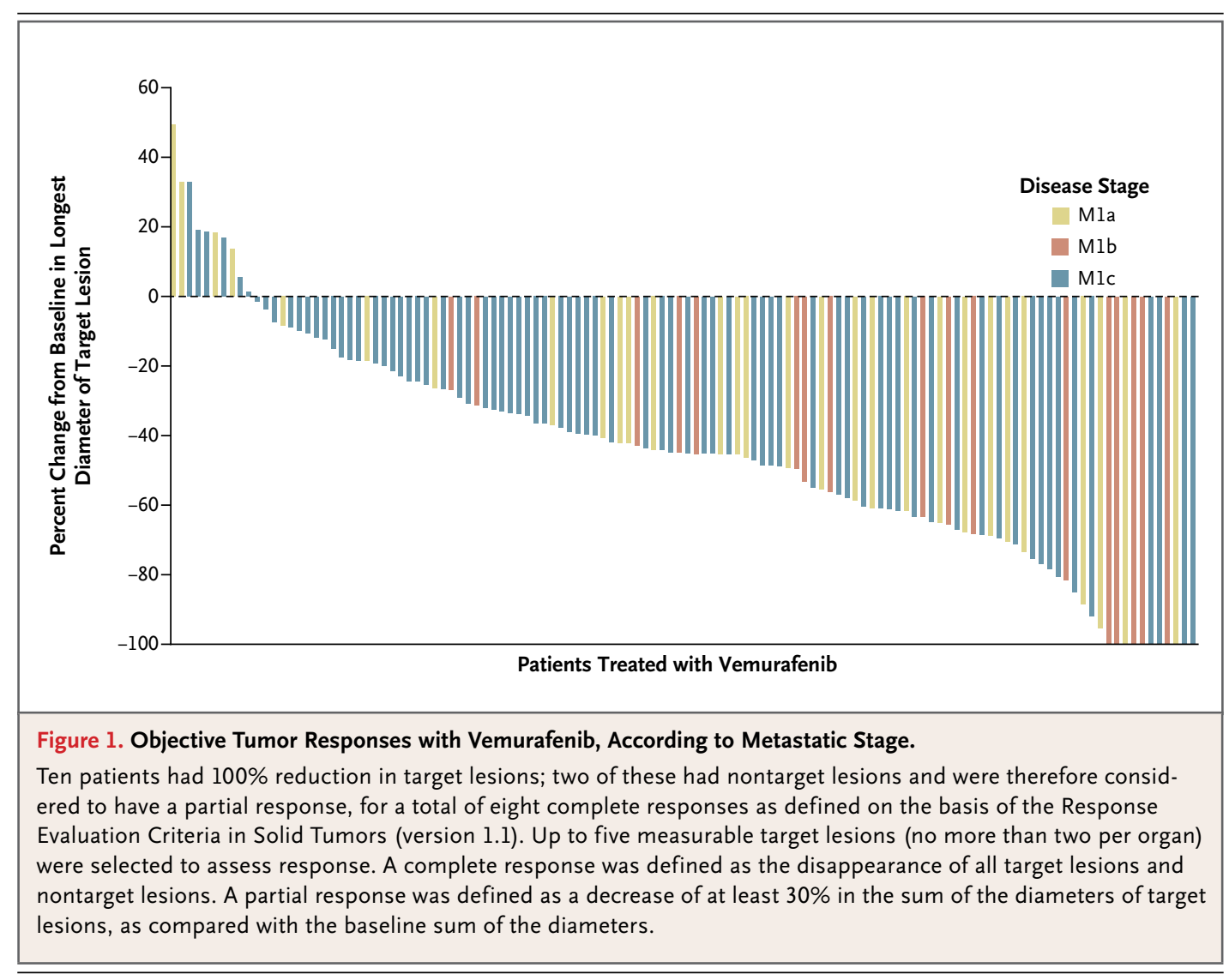

We planned to enroll at least 90 patients, for a sample size of at least 80 patients who could be evaluated, to demonstrate that if the observed overall response rate were greater than $30 \%$, the lower boundary of the corresponding exact two-sided $95 \%$ confidence interval would be greater than $20 \%$. The durations of response, progression-free survival, and overall survival were estimated by means of the Kaplan-Meier method, and medians with corresponding two-sided $95 \%$ confidence intervals were calculated with the use of the method of Brookmeyer and Crowley. ${ }^{21}$ Planned analyses of the IRC-assessed overall response rates were summarized for subgroups based on sex, age, lactate dehydrogenase (LDH) level, ECOG performance status, metastasis stage at the time of treatment, prior therapy, and previous treatment with interleukin-2. Subgroup analysis did not include prior therapy with ipilimumab, because few patients received ipilimumab before enrollment. An additional independent statistical review of all outcome data was performed by two academic statistician authors to validate all results of the industry statistician author.

\section{RESULTS}

Between October 2009 and March 2010, a total of 344 patients were screened for study entry at 13 centers (10 in the United States and 3 in Australia). Overall, 328 patients had tumor tissue tested for BRAF V600 mutations, and 184 (56\%) tested positive (Fig. A in the Supplementary Appendix). The most common reason for exclusion was a negative test for BRAF V600 (in 143 patients), followed by the presence of central nervous system metastases (in 23 patients) on brain screening by means of MRI or CT. A total of 132 patients received the study drug and made up the intention-to-treat population. We enrolled more patients than the 90 originally planned because at the time the enrollment target was met, additional patients were already being screened; they were subsequently enrolled if determined to be eligible. At the efficacy data cutoff date (July 1, 2011), the median follow-up was 12.9 months (range, 0.6 to 20.1). At the safety data cutoff date (January 31, 2011), the median follow-up was 10.4 months (range, 0.6 to 14.7$)$. 


\section{PATIENT CHARACTERISTICS}

Of the 132 patients enrolled and treated in the study, the majority were men under 65 years of age with stage M1c disease (Table 1). In all, 49\% of patients had an elevated LDH level. Only 7 patients had received prior anti-CTLA4 therapy; 1 other had a history of stable brain metastases.

\section{MOLECULAR TESTING}

After screening for BRAF V600E by means of PCRbased testing, a BRAF V600E mutation was confirmed by Sanger sequencing or pyrosequencing in 122 patients, and BRAF V600K mutations were identified in the remaining 10 patients (Fig. B in the Supplementary Appendix).

\section{EFFICACY}

According to the IRC, a complete response was achieved in 8 patients (6\%) and a partial response in 62 patients (47\%), for an overall response rate of $53 \%$ (95\% confidence interval [CI], 44 to 62) (Fig. 1 , and Fig. $\mathrm{C}$ in the Supplementary Appendix). The rate of stable disease was $29 \%$ (in 38 of the 132 patients; 95\% CI, 21 to 37). Six patients had missing assessments or data that were not able to be assessed. Only 18 patients (14\%; 95\% CI, 8 to 21 ) had primary progressive disease (Fig. $\mathrm{C}$ in the Supplementary Appendix). The investigator-assessed overall response rate was $57 \%$ (partial response, $52 \%$ of patients; complete response, $5 \%$ ), representing an $83 \%$ concordance with the IRC assessments. In all the predefined subgroups comprising more than 25 patients, the overall response rate was greater than $30 \%$, meeting the target rate in the protocol (Fig. D in the Supplementary Appendix). Patients with an $\mathrm{LDH}$ level more than 1.5 times the upper limit of the normal range had an overall response rate of 33\% (15 of 46 patients; 95\% CI, 19 to 48) - the lowest among the subgroups. Among the 10 patients with BRAF V600K mutations, 4 had a partial response, 3 had stable disease, 2 had progressive disease, and 1 had data that could not be assessed.

Twenty-three of 70 patients with a response had a maintained response at the date of efficacy data cutoff (July 1, 2011). The median duration of response according to the IRC was 6.7 months ( $95 \%$ CI, 5.6 to 8.6) (Fig. 2). Most objective responses were evident at the time of the first set of scans (week 6), but in some patients, responses did not appear until the patient had been receiving the drug for more than 6 months. Thirty-three

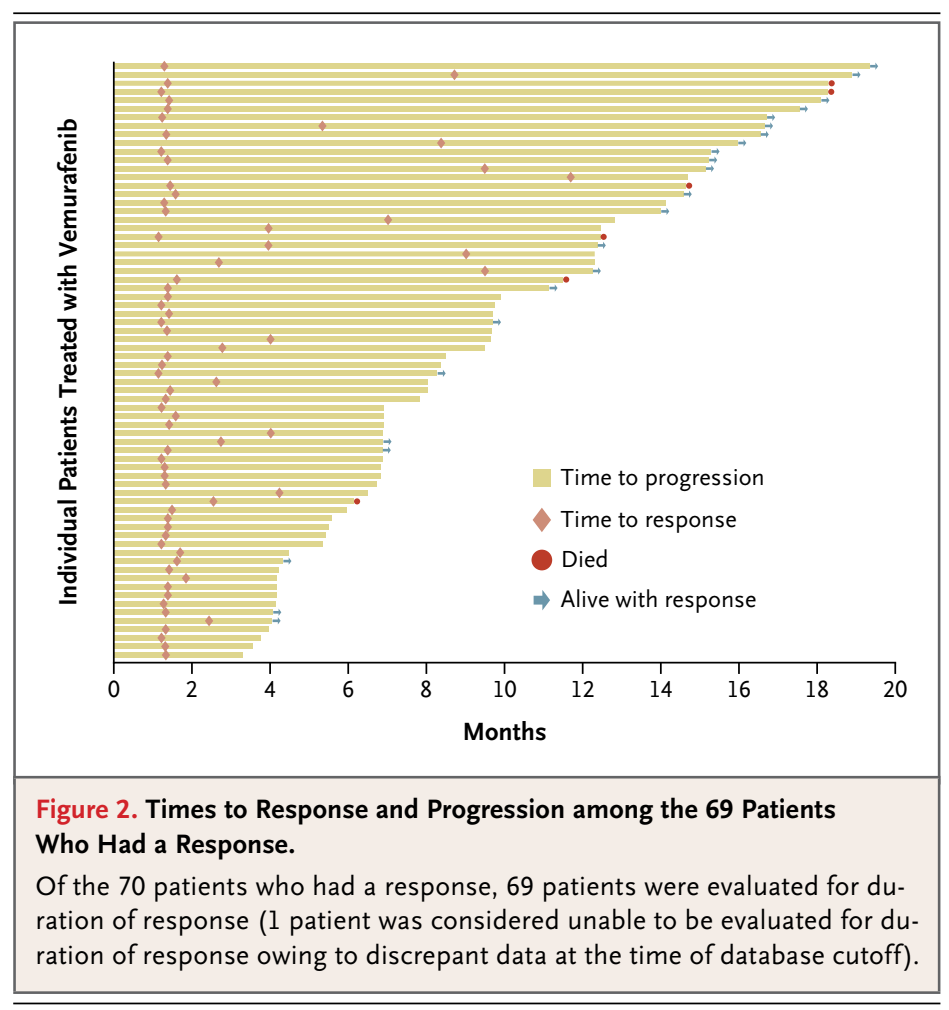

of the 132 patients $(25 \%)$ were progression-free at the time of the data cutoff. The median progression-free survival, as assessed by the IRC, was 6.8 months (95\% CI, 5.6 to 8.1). The 6-month progression-free survival rate was 56\% (95\% CI, 47 to 64) (Fig. 3A).

Of the 132 patients enrolled in the study, 62 (47\%) were alive as of July 1, 2011, and the median overall survival was 15.9 months (95\% CI, 11.6 to 18.3) (Fig. 3B). The overall survival rate at 6 months was $77 \%$ (95\% CI, 70 to 85 ), 58\% at 12 months (95\% CI, 49 to 67), and estimated to be $43 \%$ at 18 months (95\% CI, 33 to 53). During the followup period, 32 patients (24\%) received ipilimumab after they had disease progression while receiving vemurafenib. In an unplanned post hoc analysis, median overall survival remained at 15.9 months (95\% CI, 8.0 to not reached) even when these 32 patients were not included.

\section{SAFETY}

The safety data cutoff was January 31, 2011. Most patients had at least one adverse event related to the study drug (Table 2). The most commonly reported adverse events were arthralgia, rash, a photosensitivity reaction, fatigue, and alopecia. Several patients had asymptomatic, transient elevations in 

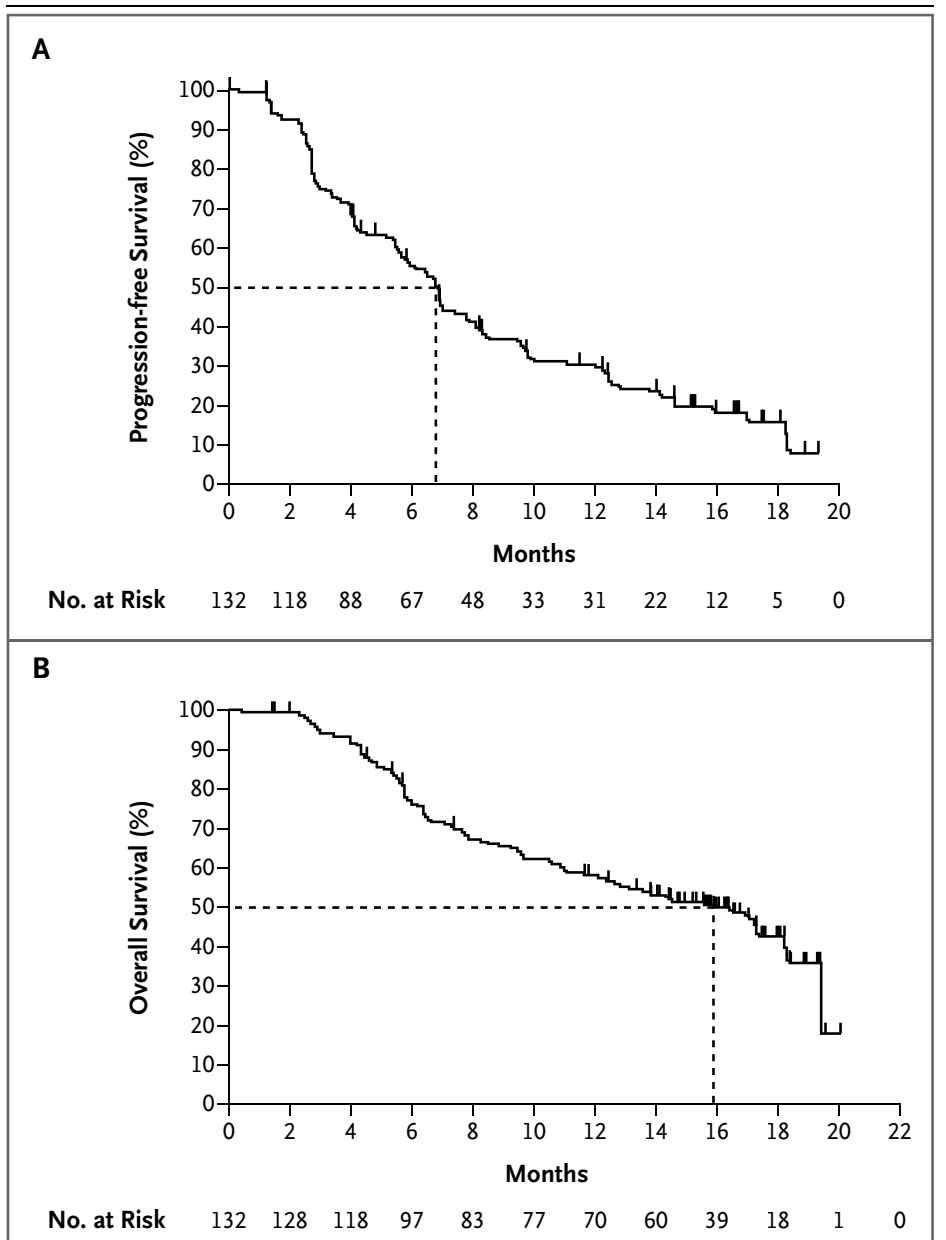

Figure 3. Kaplan-Meier Estimates of Progression-free and Overall Survival. Shown is the percentage of vemurafenib-treated patients with progressionfree survival (Panel A) and overall survival (Panel B). The dashed lines indicate the medians. Tick marks along the curves represent the date of the last tumor assessment before the time of data cutoff among patients with censored data without disease progression or death. liver-enzyme levels. Four patients discontinued treatment because of adverse events, including retinal-vein occlusion in one. One patient died owing to rapid progression of melanoma and acute renal failure, possibly related to the study drug. Three patients had transient palsies of the seventh cranial nerve (with one patient having both synchronous and bilateral palsies). After resolution, these patients were able to resume vemurafenib.

Fifty-nine (45\%) of the 132 patients had their dose reduced; dose interruptions were required in 85 patients (64\%). The adverse events that most frequently led to dose modification or interruption included rash, arthralgia, elevated liver-enzyme levels, and photosensitivity reactions. Patients received a median dose of $1740 \mathrm{mg}$ per day, which represents $91 \%$ of the intended dose of $1920 \mathrm{mg}$ per day.

Development of cutaneous squamous-cell carcinoma or keratoacanthoma was reported in 34 patients (26\%), typically consisting of only one lesion (in 20 patients) or two lesions (in 6 patients). Three lesions developed in 4 other patients, and four, five, six, and seven lesions developed in 1 patient each. The median time to development of the first cutaneous squamous-cell carcinoma or keratoacanthoma lesion was 8 weeks (range, 2 to 36) (Fig. $\mathrm{E}$ in the Supplementary Appendix). On central pathological review, 39 of 43 cutaneous squamouscell carcinoma lesions were either keratoacanthoma or mixed keratoacanthoma type; the remaining 4 were invasive cutaneous squamous-cell carcinoma. Eight cases of basal-cell carcinoma were identified. No mucosal squamous-cell carcinoma or metastases of cutaneous squamous-cell carcinoma were observed.

\section{DISCUSSION}

Treatment options for patients with advanced melanoma are limited. ${ }^{22}$ Since its discovery in 2002, the BRAF V600E mutant kinase has been considered a promising therapeutic target for this disease. The previously reported phase 1 study with vemurafenib in patients with BRAF V600-mutant metastatic melanoma provided evidence that inhibition of the oncogenic MAPK pathway resulted in significant antitumor activity. ${ }^{15,23}$ The major objective of the current phase 2 clinical trial was to define, in a larger number of patients, the overall response rate with vemurafenib in advanced melanoma. We report a confirmed response rate of over $50 \%$ in patients with previously treated metastatic melanoma bearing the V600E or V600K BRAF mutations. Most responses were rapid, with less than $15 \%$ of patients having had disease progression at their first evaluation. Therefore, this trial shows that vemurafenib has clinically evident antitumor activity in metastatic melanoma and that response rates are higher than those associated with previously used treatments. ${ }^{1-8,22}$ Reanalysis of the response rate in the phase 1 study according to the response criteria used in our phase 2 study yielded a similar overall response rate of $56 \%$. Furthermore, although the median durations of response and progression-free survival were less than 
7 months, some responses were delayed, with one fourth of patients remaining progression-free after a median follow-up period of 13 months. The median overall survival was nearly 16 months in this group of patients with melanomas expressing the relevant mutation. The patients did not have favorable baseline characteristics $(61 \%$ with stage M1c disease and 49\% with elevated LDH level) as compared with those in other large, phase 2 and phase 3 studies of melanoma. In fact, the BRAF mutation has been associated with shortened survival in patients with metastatic disease. ${ }^{24}$ The long median overall survival was not simply due to post-progression ipilimumab use in some patients, because exclusion of these patients from the analysis did not change the median overall survival.

Toxic effects were common but not severe or life-threatening in most instances. Although some patients required dose interruptions or reductions, patients were able to receive most of their intended daily dose. The toxic effects were largely related to the skin. As reported previously, the BRAF inhibitor vemurafenib, as with other RAF inhibitors, is associated with the development of cutaneous squamous-cell carcinoma or keratoacanthoma but not squamous-cell carcinoma derived from other organs. ${ }^{15,25,26}$ Lesions usually manifested in the first 8 to 12 weeks of treatment and were effectively managed with simple resection without discontinuation of vemurafenib. This suggests differential effects of vemurafenib on cells without oncogenic BRAF. Preclinical models have shown that BRAF inhibitors can paradoxically enhance activation of the MAPK pathway in cancer cells with wild-type BRAF that carry upstream RAS mutations. $^{27-30}$ This mechanism may play a role in the development of cutaneous squamous-cell carcinomas. ${ }^{30}$

As with most targeted therapies that block a driver oncogene, cancer cells can develop acquired resistance with continuous dosing. The molecular mechanisms of vemurafenib resistance are under investigation. The currently available data suggest that reactivation of the MAPK pathway through the emergence of truncated hyperactive forms of BRAF, ${ }^{31}$ secondary mutations in NRAS (the neuroblastoma RAS viral oncogene homologue) ${ }^{32}$ or MEK (MAP kinase kinase), ${ }^{33}$ up-regulation of COT (also known as TPL2 or MAP3K8), ${ }^{34}$ or activation of alternative survival pathways induced by increased expression of receptor tyrosine kinases but

\begin{tabular}{|lccc|}
\hline Table 2. Adverse Events Related to the Study Drug. \\
\hline Adverse Event & Any Grade & Grade 3 & Grade 4 \\
& \multicolumn{3}{c|}{ number (percent) } \\
Total & $130(98)$ & $79(60)$ & $5(4) *$ \\
Arthralgia & $78(59)$ & $8(6)$ & - \\
Rash & $69(52)$ & $9(7)$ & - \\
Photosensitivity reaction & $69(52)$ & $4(3)$ & - \\
Fatigue & $56(42)$ & $2(2)$ & - \\
Alopecia & $48(36)$ & - & - \\
Pruritus & $38(29)$ & $3(2)$ & - \\
Skin papilloma & $38(29)$ & - & - \\
Cutaneous squamous-cell carcinoma & $34(26)$ & $34(26)$ & - \\
$\quad$ or keratoacanthoma† & & & - \\
Nausea & $30(23)$ & $2(2)$ & - \\
Elevated liver enzymes & $23(17)$ & $8(6) \ddagger$ & $4(3) \mathbb{\int}$ \\
Peripheral neuropathy & $13(10)$ & $1(1)$ & - \\
Palmar-plantar erythrodysesthesia & $13(10)$ & $2(2)$ & - \\
Facial palsy & $3(2)$ & $1(1)$ & - \\
Hyperuricemia & $3(2)$ & - & $1(1)$ \\
Retinal-vein occlusion & $1(1)$ & $1(1) \mathbb{}$ & - \\
Delirium & $1(1)$ & $1(1) \sqrt{ }$ & - \\
\hline
\end{tabular}

* One patient had two grade 4 adverse events.

$\uparrow$ Cases of cutaneous squamous-cell carcinoma or keratoacanthoma were generally managed with simple excision and did not usually require dose modification.

$\Varangle$ Grade 3 elevated liver-enzyme levels were managed by reducing the dose of the study drug; one affected patient was removed from the study.

$\int$ Grade 3 or 4 adverse events led to discontinuation of therapy.

not by secondary point mutations in BRAF ${ }^{32,35}$ are all mechanisms of resistance. Elucidating approaches that can overcome or prevent acquired resistance is critical to further advances in the treatment of melanoma.

In conclusion, this trial shows a high rate of response to vemurafenib in patients with metastatic melanoma and activating BRAF mutations. These results independently confirm the high response rate and response duration shown in a phase 1 trial. The long follow-up period in our study provides critical information on long-term overall survival, not yet shown in the phase 3 trial comparing vemurafenib with dacarbazine. ${ }^{19}$ Targeted therapy aimed at oncogenic BRAF V600 induces responses in half the patients and a median survival of 16 months.

Supported by Hoffmann-La Roche.

Disclosure forms provided by the authors are available with the full text of this article at NEJM.org. 
We thank the study team (including research nurses and data coordinators), sponsor members (Stephanie Kapsalis, Stuart Teller, Raniya Kassem, Suzanne Cheng, Kerstin Trunzer, Axel Muehling, and Mark Rothe), and additional investigators (Lance Cowey at Baylor Sammons Cancer Center, Leslie Fecher at Abramson Cancer Center, John A. Glaspy at Jonsson Comprehen- sive Cancer Center, F. Stephen Hodi at Dana-Farber Cancer Institute, Damien Kee at Peter MacCallum Cancer Centre, Georgina V. Long at Westmead Institute for Cancer Research, and David McDermott at Beth Israel Deaconess Medical Center) for their contributions to this study, and we appreciate the commitment of all the patients who were screened for and enrolled in this trial.
REFERENCES

1. Tsao H, Atkins MB, Sober AJ. Management of cutaneous melanoma. $\mathrm{N}$ Engl J Med 2004;351:998-1012. [Erratum, N Engl J Med 2004;351:2461.]

2. Chapman PB, Einhorn LH, Meyers ML, et al. Phase III multicenter randomized trial of the Dartmouth regimen versus dacarbazine in patients with metastatic melanoma. J Clin Oncol 1999;17:2745-51. 3. Atkins $M B$, Hsu J, Lee $S$, et al. Phase III trial comparing concurrent biochemotherapy with cisplatin, vinblastine, dacarbazine, interleukin-2, and interferon alfa-2b with cisplatin, vinblastine, and dacarbazine alone in patients with metastatic malignant melanoma (E3695): a trial coordinated by the Eastern Cooperative Oncology Group. J Clin Oncol 2008;26:5748-54.

4. Middleton MR, Grob JJ, Aaronson N, et al. Randomized phase III study of temozolomide versus dacarbazine in the treatment of patients with advanced metastatic malignant melanoma. J Clin Oncol 2000; 18:158-66. [Erratum, J Clin Oncol 2000; 18:2351.]

5. Falkson CI, Ibrahim J, Kirkwood JM, Coates AS, Atkins MB, Blum RH. Phase III trial of dacarbazine versus dacarbazine with interferon alpha-2b versus dacarbazine with tamoxifen versus dacarbazine with interferon alpha-2b and tamoxifen in patients with metastatic malignant melanoma: an Eastern Cooperative Oncology Group study. J Clin Oncol 1998;16:1743-51. 6. Atkins MB, Lotze MT, Dutcher JP, et al. High-dose recombinant interleukin 2 therapy for patients with metastatic melanoma: analysis of 270 patients treated between 1985 and 1993. J Clin Oncol 1999;17:2105-16.

7. Hodi FS, O'Day SJ, McDermott DF, et al. Improved survival with ipilimumab in patients with metastatic melanoma. N Engl J Med 2010;363:711-23. [Erratum, N Engl J Med 2010;363:1290.]

8. Robert C, Thomas L, Bondarenko I, et al. Ipilimumab plus dacarbazine for previously untreated metastatic melanoma. N Engl J Med 2011;364:2517-26.

9. Davies H, Bignell GR, Cox C, et al. Mutations of the BRAF gene in human cancer. Nature 2002;417:949-54.

10. Gray-Schopfer V, Wellbrock C, Marais R. Melanoma biology and new targeted therapy. Nature 2007;445:851-7.

11. Tsai J, Lee JT, Wang W, et al. Discovery of a selective inhibitor of oncogenic
B-Raf kinase with potent antimelanoma activity. Proc Natl Acad Sci U S A 2008; 105:3041-6.

12. Sфndergaard JN, Nazarian R, Wang $\mathrm{Q}$, et al. Differential sensitivity of melanoma cell lines with BRAFV600E mutation to the specific Raf inhibitor PLX4032. J Transl Med 2010;8:39.

13. Yang H, Higgins B, Kolinsky K, et al. RG7204 (PLX4032), a selective BRAFV600E inhibitor, displays potent antitumor activity in preclinical melanoma models. Cancer Res 2010;70:5518-27. [Erratum, Cancer Res 2010;70:9527.]

14. Joseph EW, Pratilas CA, Poulikakos PI, et al. The RAF inhibitor PLX4032 inhibits ERK signaling and tumor cell proliferation in a V600E BRAF-selective manner. Proc Natl Acad Sci U S A 2010; 107:14903-8.

15. Flaherty KT, Puzanov I, Kim KB, et al. Inhibition of mutated, activated BRAF in metastatic melanoma. N Engl J Med 2010; 363:809-19.

16. Chapman PB, Hauschild A, Robert C, et al. Improved survival with vemurafenib in melanoma with BRAF V600E mutation. N Engl J Med 2011;364:2507-16.

17. Oken MM, Creech RH, Tormey DC, et al. Toxicity and response criteria of the Eastern Cooperative Oncology Group. Am J Clin Oncol 1982;5:649-55.

18. Thomas RK, Nickerson E, Simons JF et al. Sensitive mutation detection in heterogeneous cancer specimens by massively parallel picoliter reactor sequencing. Nat Med 2006;12:852-5. [Erratum, Nat Med 2006;12:1220.]

19. Eisenhauer EA, Threasse P, Bogaerts $\mathrm{J}$, et al. New response criteria in solid tumors: revised RECIST guideline (version 1.1). Eur J Cancer 2009;45:228-47.

20. Clopper CJ, Pearson ES. The use of confidence or fiducial limits illustrated in the case of the binomial. Biometrika 1934 26:404-3.

21. Brookmeyer R, Crowley J. A confidence interval for the median survival time. Biometrics 1982;38:29-41.

22. Korn EL, Liu PY, Lee SJ, et al. Metaanalysis of phase II cooperative group trials in metastatic stage IV melanoma to determine progression-free and overall survival benchmarks for future phase II trials. J Clin Oncol 2008;26:527-34.

23. Bollag G, Hirth P, Tsai J, et al. Clinical efficacy of a RAF inhibitor needs broad target blockade in BRAF-mutant melanoma. Nature 2010;467:596-9.

24. Long GV, Menzies AM, Nagrial AM, et al. Prognostic and clinicopathologic associations of oncogenic BRAF in metastatic melanoma. J Clin Oncol 2011;29:1239-46. 25. Lacouture ME, McArthur GA, Chapman PB, et al. PLX4032 (RG7204), a selective mutant RAF inhibitor: clinical and histologic characteristics of therapy-associated cutaneous neoplasms in a phase I trial. J Clin Oncol 2010;28:Suppl:15S. abstract. 26. Robert C, Arnault JP, Mateus C. RAF inhibition and induction of cutaneous squamous cell carcinoma. Curr Opin Oncol 2011;23:177-82.

27. Heidorn SJ, Milagre C, Whittaker S, et al. Kinase-dead BRAF and oncogenic RAS cooperate to drive tumor progression through CRAF. Cell 2010;140:209-21.

28. Hatzivassiliou G, Song K, Yen I, et al. RAF inhibitors prime wild-type RAF to activate the MAPK pathway and enhance growth. Nature 2010;464:431-5.

29. Poulikakos PI, Zhang C, Bollag G, Shokat KM, Rosen N. RAF inhibitors transactivate RAF dimers and ERK signalling in cells with wild-type BRAF. Nature 2010;464:427-30.

30. Su F, Viros A, Milagre C, et al. RAS mutations in cutaneous squamous-cell carcinomas in patients treated with BRAF inhibitors. N Engl J Med 2012;366:207-15. 31. Poulikakos PI, Persaud Y, Janakiraman $\mathrm{M}$, et al. RAF inhibitor resistance is mediated by dimerization of aberrantly spliced BRAF(V600E). Nature 2011;480: 387-90.

32. Nazarian R, Shi H, Wang Q, et al. Melanomas acquire resistance to B-RAF(V600E) inhibition by RTK or N-RAS upregulation. Nature 2010;468:973-7.

33. Wagle N, Emery C, Berger MF, et al. Dissecting therapeutic resistance to RAF inhibition in melanoma by tumor genomic profiling. J Clin Oncol 2011;29:3085-96.

34. Johannessen CM, Boehm JS, Kim SY, et al. COT drives resistance to RAF inhibition through MAP kinase pathway reactivation. Nature 2010;468:968-72.

35. Villanueva J, Vultur A, Lee JT, et al. Acquired resistance to BRAF inhibitors mediated by a RAF kinase switch in melanoma can be overcome by cotargeting MEK and IGF-1R/PI3K. Cancer Cell 2010; 18:683-95.

Copyright (c) 2012 Massachusetts Medical Society. 